\title{
Erratum to: Cypermethrin exposure during puberty disrupts testosterone synthesis via downregulating StAR in mouse testes
}

Hua Wang • Qun Wang • Xian-Feng Zhao • Ping Liu •

Xiu-Hong Meng · Tao Yu • Yan-Li Ji • Heng Zhang •

Cheng Zhang $\cdot$ Ying Zhang $\cdot$ De-Xiang Xu

Published online: 12 December 2009

(C) Springer-Verlag 2009

Erratum to: Arch Toxicol

DOI 10.1007/s00204-009-0479-y

Unfortunately there is a small mistake in Fig. 4a: " $\beta$-actin" should read "GAPDH".

The online version of the original article can be found under doi:10.1007/s00204-009-0479-y.

H. Wang $\cdot$ Q. Wang $\cdot$ X.-F. Zhao $\cdot$ P. Liu $\cdot$ X.-H. Meng · T. Yu

Y.-L. Ji $\cdot$ H. Zhang $\cdot$ C. Zhang $\cdot$ Y. Zhang $\cdot$ D.-X. Xu ( $\)$

Department of Toxicology, Anhui Medical University,

Hefei 230032, China

e-mail: xudex@mail.hf.ah.cn 